\title{
Multi-kilometer Long, Longitudinally Uniform Hollow Core Photonic Bandgap Fibers for Broadband Low Latency Data Transmission
}

\author{
Yong Chen, Member, OSA, Zhixin Liu, Member, IEEE, Seyed R. Sandoghchi, Member, IEEE, Gregory T. Jasion, \\ Tom D. Bradley, Eric Numkam Fokoua, John R. Hayes, Natalie V. Wheeler, David R. Gray, Member, OSA, \\ Brian J. Mangan, Radan Slavík, Senior Member, IEEE, Member, OSA, Francesco Poletti, Member, IEEE, \\ Marco N. Petrovich, Senior Member, IEEE, Senior Member, OSA, \\ and David J. Richardson, Fellow, IEEE, Fellow, OSA
}

(Post-Deadline)

\begin{abstract}
The low intrinsic nonlinearity and low signal latency characteristic of Hollow Core Photonic Bandgap Fibers (HCPBGFs) have fueled strong interest for data transmission applications. Whereas most research to date has looked at improving the optical performance of HC-PBGFs (e.g., reducing the loss, increasing the transmission bandwidth and achieving well-tempered modal properties through the suppression of surface mode resonances). In this study, we address the challenging problem of scaling up the fabrication of these fibers to multi-kilometer lengthsan indispensable step to prove this fiber technology as viable. We report the fabrication of low loss, wide bandwidth HC-PBGFs operating both in the conventional telecoms window $(1.55 \mu \mathrm{m})$ and in the predicted region of minimum loss $(2 \mu \mathrm{m})$, in lengths that substantially exceed the state of the art. At $2 \mu \mathrm{m}$, we obtained a $3.85 \mathrm{~km}$ long fiber with $\approx 3 \mathrm{~dB} / \mathrm{km}$ loss and $>160 \mathrm{~nm}$ wide $3 \mathrm{~dB}$ bandwidth. Additionally, we report an HC-PBGF operating at $1.55 \mu \mathrm{m}$ with a length of just over $11 \mathrm{~km}$, transmission bandwidth in excess of $200 \mathrm{~nm}$ and a longitudinally uniform loss of $\approx 5 \mathrm{~dB} / \mathrm{km}$, measured via cutback and an integrated scattering method. We used the latter fiber to demonstrate error-free, low-latency, direct-detection $10 \mathrm{~Gb} / \mathrm{s}$ transmission across the entire $\mathrm{C}$-Band as well as $20 \mathrm{~Gb} / \mathrm{s}$ quadrature phase shift keyed transmission. These represent the first demonstrations of data transmission over a length of $\mathrm{HC}$ PBGF exceeding $10 \mathrm{~km}$.
\end{abstract}

Index Terms-Fiber communications, hollow core, low latency, microstructured optical fibers, photonic bandgap fibers.

Manuscript received June 23, 2015; revised August 24, 2015; accepted August 25, 2015. Date of publication September 2, 2015; date of current version January 24, 2016. This work was supported in part by EU FP7 through project MODE-GAP under Grant 258033 and in part by the U.K. Engineering and Physical Sciences Research Council (EPSRC) under Grant EP/H02607X/1 (Centre for Innovative Manufacturing in Photonics) and Grant EP/I061196X (HYPERHIGHWAY).

Y. Chen, Z. Liu, S. R. Sandoghchi, G. Jasion, T. D. Bradley, E. Numkam, J. R. Hayes, N. V. Wheeler, D. R. Gray, R. Slavík, F. Poletti, M. N. Petrovich, and D. J. Richardson are with the Optoelectronics Research Centre, University of Southampton, Southampton SO17 1BJ, U.K. (e-mail: yc1m12@soton. ac.uk; z.liu@soton.ac.uk; srs1g12@orc.soton.ac.uk; G.Jasion@soton.ac.uk; T.Bradley@soton.ac.uk; ernf1g10@ orc.soton.ac.uk; jrh@orc.soton.ac.uk; nvw1v10@orc.soton.ac.uk; drg1v07@orc.soton.ac.uk; r.slavik@soton.ac.uk frap@orc.soton.ac.uk; mnp@orc.soton.ac.uk; djr@orc.soton.ac.uk).

B. J. Mangan is with the OFS Labs, Somerset, NJ 08873 USA (e-mail: bmangan@ofsoptics.com).

Color versions of one or more of the figures in this paper are available online at http://ieeexplore.ieee.org.

Digital Object Identifier 10.1109/JLT.2015.2476461

\section{INTRODUCTION}

$\mathbf{T}$ HERE has been a resurgence in interest in recent years in the use of Hollow Core Photonic Bandgap Fibers (HCPBGFs) for telecommunication applications [1]. The primary driver is the potential for low-latency, guided-wave data transmission which is of huge interest in numerous time-sensitive application sectors including: intra- and inter- data center interconnection, high performance computing, large-scale high energy physics experiments, and for bespoke data networks for the financial sector in particular. For all these applications, the $1.54 \mu \mathrm{s} / \mathrm{km}$ latency reduction [2] offered by HC-PBGFs may represent a decisive advantage over existing and competing fiber technologies.

Remarkable progress has been attained in recent years in the development of these complex fiber waveguides. Whilst the unique enabling properties of HC-PBGFs, such as their low latency [2], low nonlinearity [3], low thermal sensitivity [4] and high radiation hardness [5] have been reliably confirmed, along with their ability to support broad-bandwidth (BW) high capacity transmission on the fundamental [6] as well as on multiple optical modes [7], most studies to date have used fiber samples of relatively modest lengths (typically less than $1 \mathrm{~km}$ ). This has primarily been due to the difficulties in scaling up the fabrication procedures that are used to make such fibers (typically based on the so-called "stack-and-draw" method) [1], and in eliminating defects/contamination that can occur during preform manufacture [8], and also due to the lack of means to ensure that a sufficient structural uniformity is maintained along the full fiber draw. The demonstration that HC-PBGF can be reliably fabricated in long (multi-km) lengths has therefore emerged as one of the highest research priorities. Until long fiber samples become available to test in application trials of realistic scale, industrial and commercial interest in the technology will obviously remain limited.

In this paper, exploiting an improved understanding deriving from various new numerical and experimental fabrication and characterization tools that we have developed over the past few years, we report a significant step forward in HC-PBGF development. In particular, we have achieved what we believe to be 
the first high performance HC-PBGF with a length in excess of $10 \mathrm{~km}$, providing a combination of transmission $\mathrm{BW}(>200 \mathrm{~nm})$ and loss $(\approx 5 \mathrm{~dB} / \mathrm{km})$ that is close to the current state-of-the-art for much shorter fiber lengths. The longest fiber span obtained $(11 \mathrm{~km})$ was designed for operation at conventional telecom wavelengths $(1.55 \mu \mathrm{m})$, however we also obtained multi-km long fiber spans operating at the wavelength of minimum loss for HC-PBGFs, i.e., $\approx 2 \mu \mathrm{m}$ [9], [10]. In this instance, the longest fiber sample obtained was $3.85 \mathrm{~km}$, which represents a $3.3 \times$ improvement in length for a HC-PBGF operating in this waveband [11]. A potential new "telecom window" at $2 \mu \mathrm{m}$ would benefit from the recently developed thulium-doped fiber amplifier which offers gain BW greatly exceeding that of traditional erbium-doped fiber amplifiers at $1.55 \mu \mathrm{m}$ [12].

In addition, we utilized the $11 \mathrm{~km}$ long HC-PBGF — a length compatible with many of the applications detailed above-to demonstrate the viability of $10 \mathrm{Gbit} / \mathrm{s}$ data transmission using direct detection with a simple return-to-zero ON-OFF keying (RZ-OOK) modulation and $20 \mathrm{~Gb} / \mathrm{s}$ using the quadrature phase shift keying (QPSK) coherent modulation format. The propagation latency reduction in this fiber span as compared to a similar length of standard single mode transmission fiber (e.g., SMF28 or any non-zero dispersion shifted fiber) is confidently estimated to be in excess of $15 \mu \mathrm{s}$.

\section{HC-PBGF FABRICATION UPSCALING CHALLENGES}

HC-PBGFs are characterized by having a very complex structure and optical properties that are strongly dependent upon its fine details. Consequently, their fabrication, based on the stackand-draw method, is substantially more involved as compared to that of conventional solid fibers. HC-PBGFs are obtained by manually assembling stacks composed of a few hundred capillaries with $\approx$ mm diameter and a high geometrical consistency (at the $\approx \mu \mathrm{m}$ level); these are tightly packed and fused inside a glass jacketing tube and drawn into fiber using a two-step procedure [1]. Careful control of the pressure inside the holes (to $\approx 0.1 \mathrm{kPa}$ ) is paramount to tune the fiber structure to the target design and to maintain longitudinal consistency during the final fiber draw down step. Stack-and-draw is versatile (the capillary elements can be stacked in a wide range of geometries) and is at least partially amenable to automation; on the other hand, the process is rather slow and requires extensive manual handling, which often correlates with a higher likelihood of introducing stacking errors, defects and contamination within the preforms. The presence of defects and/or contamination within the preforms in turn leads to a higher fiber failure rate during the second stage drawing process, making it very challenging in practice to obtain long fiber lengths. Indeed, despite several groups worldwide attempting to improve HC-PBGF fabrication for several years, until very recently only a handful of reports of fiber spans of the order of $\approx 1 \mathrm{~km}$ had been reported in the literature [11], [13], [14]. Whilst conventional fibers are often fabricated at low tension in order to alleviate the incidence of fiber breakages and to maintain the integrity of the fiber over extremely long lengths of drawn fiber, this is often a less viable option for HC-PBGFs where it is imperative to minimize any structural distortions, which are promoted by a higher tempera- ture/low tension drawing regime. HC-PBGFs are thus typically drawn at high tension $(>200 \mathrm{~g})$. It is therefore clear that the ability to produce first and second stage preforms as free as possible of contamination (e.g., by assembling stack in a clean, controlled environment) is of crucial importance. We have not only modified our preform assembly and processing to achieve this goal, but we also have applied a range of techniques, such as X-ray computational tomography, for the purpose of non-destructively investigating the quality of our preforms [8]. Furthermore, the presence of longitudinal inconsistencies in the fiber structure ("defects"), even of small amplitude and/or short characteristic length scale, may potentially lead to a significant worsening of the fiber's performance, for instance through increased scattering, by introducing undesirable coupling between optical modes supported within the core, or also by introducing surface modes at the defect point. These effects narrow the achievable BW, increase the transmission loss, and cause ill-controlled modal properties and excitation, and should obviously be avoided. We have previously presented the results of a comprehensive investigation of such defects in some of our fibers [15] and spent considerable effort in trying to link them to defects in the corresponding preform, to understand their origin and eliminate them.

Upscaling the fiber yield requires bigger canes and preforms to be used in both drawing stages ("caning" and fiber drawing). This may imply that one of the main benefits of a two-step procedure, i.e., reducing the draw-down ratio of each step as compared to an equivalent single-step procedure, would be partially lost. A lower draw-down ratio is beneficial as it allows a higher stress to be applied at the wide end of the glass body (preform) for a certain stress at the narrow end (drawn cane or fiber), while the maximum value of the latter is limited by the tensile strength of the glass [16]. This gives the opportunity to draw in a regime (higher stress through reduced temperature or increased feed rate) in which the microstructure has more lateral viscous stiffness and can thereby resist the distortions caused by surface tension [16], [17]. However, upscaling reduces the ability to achieve a higher lateral stiffness and thus has potential to bring about distortions within the microstructure [17]. Understanding the full impact of upscaling requires the development of precise fluid dynamic models, capable of capturing the evolution along the neck down region. These tools afford a much easier exploration of the draw parameter space [18] and have been employed in association with experimental observations to help narrow down the optimized draw parameter ranges which afford a minimization of structural deformations. These models indicate that stricter criteria for the precise balance between applied pressures and surface tension during the HC-PBGFs fabrication for bigger canes and preforms. Table I summarizes the main challenges relating to upscaling the yield per draw of HC-PBGFs along with the solutions we have identified and investigated.

\section{FABRICATION OF MULTI-KILOMETER LONG HC-PBGFs}

We report two record length, wide BW HC-PBGFs operating in the spectral regions at 1.55 and $2 \mu \mathrm{m}$, respectively. Both fibers have a 19 cell core (i.e., composed of 19 missing unit cells) and 
TABLE I

HC-PBGF UPSCALING CHALLENGES

\begin{tabular}{ll}
\hline \hline Challenges & \multicolumn{1}{c}{ Solutions } \\
\hline Eliminate fibre breaks & $\begin{array}{l}\text { Optimize drawing tension - trade off with } \\
\text { distortions } \\
\text { Improve glass handling and processing } \\
\text { Improve cleanliness of stacking process }\end{array}$ \\
Minimise longitudinal defects & $\begin{array}{l}\text { Identify defects, trace them back to } \\
\text { fabrication process and eliminate } \\
\text { Increase volume of glass, } \\
\text { minimize distortions }\end{array}$ \\
$\begin{array}{l}\text { Accurate balance between pressure and } \\
\text { surface tension }\end{array}$ \\
$\begin{array}{l}\text { Fluid dynamic modelling } \\
\text { Optimization of drawing parameters via } \\
\text { "virtual draw" }\end{array}$ \\
\hline \hline
\end{tabular}

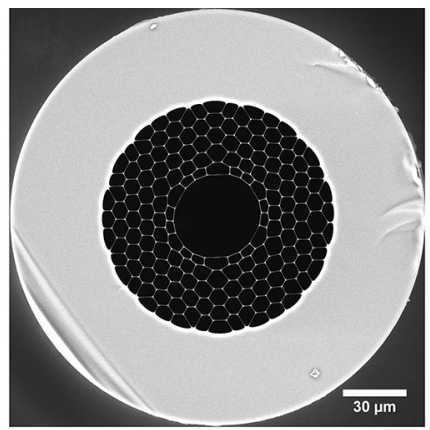

(a)

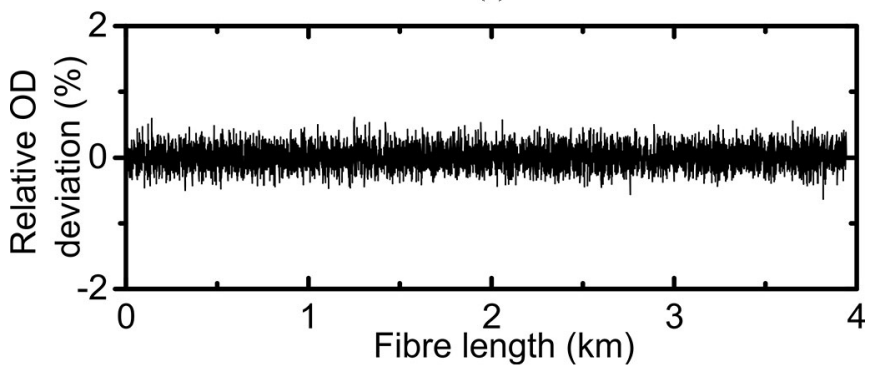

(b)

Fig. 1. (a) SEM image of the fabricated HC-PBGF operating at $2 \mu \mathrm{m}$; the fiber has a 19 cell core surrounded by five rings of cladding holes (plus an incomplete ring). (b) Diameter deviation versus fiber length measured during fiber drawing for the same fiber.

a 5 cladding ring structure, see Figs. 1(a) and Fig. 3(d), and were fabricated via a two-stage stack-and-draw technique using canes produced from the same primary stack. Through the use of a very thin core tube (supplied by OFS Labs), which allowed us to increase the length of uniform cane produced from the first stage draw, and careful preform design and process control, we successfully scaled up the yield of high quality fiber to the unprecedented lengths of $3.85 \mathrm{~km}$ at $2 \mu \mathrm{m}$ [19] and $11 \mathrm{~km}$ at $1.55 \mu \mathrm{m} \mathrm{[20].} \mathrm{In} \mathrm{order} \mathrm{to} \mathrm{minimize} \mathrm{the} \mathrm{surface} \mathrm{mode} \mathrm{issues}$ typically associated with the use of an oversized capillary tube to define the hollow core [1], we designed our primary stack to have an optimized ratio between core and cladding strut thickness. To further maximize the transmission $\mathrm{BW}$, we targeted extremely high values of air filling fraction $(\geq 96 \%)$ of the cladding.

\section{A. Fabrication of a $3.85 \mathrm{~km}$ Long HC-PBGF at $2 \mu \mathrm{m}$}

We initially targeted the spectral region around $2 \mu \mathrm{m}$, which typically involves scaling up the fiber structure as compared

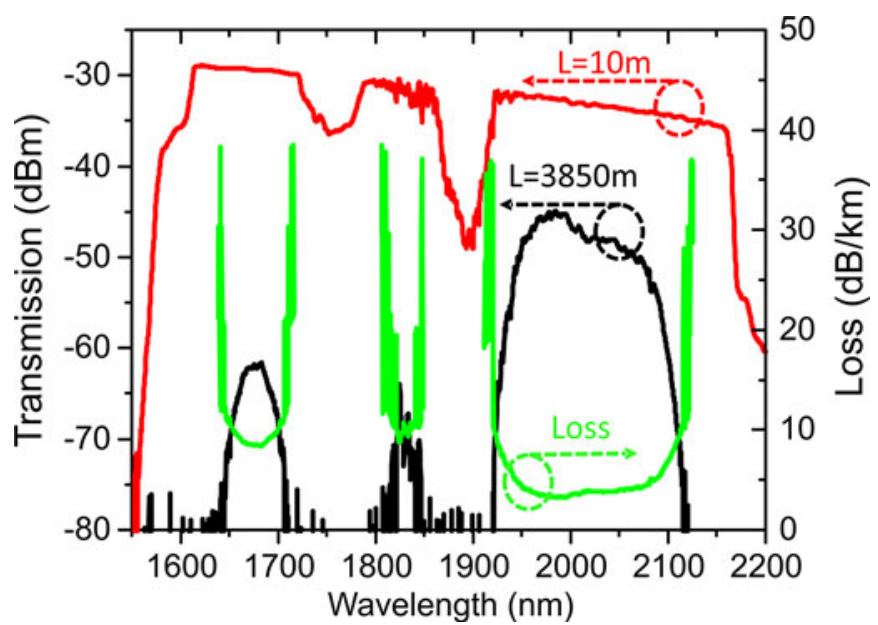

Fig. 2. Spectral attenuation of the $2 \mu \mathrm{m}$ HC-PBGF, measured from a $3.85 \mathrm{~km}$ long cutback. Note the very wide bandgap (1550-2175 nm) segmented into three windows (widest is $>160 \mathrm{~nm}$ ) by surface modes.

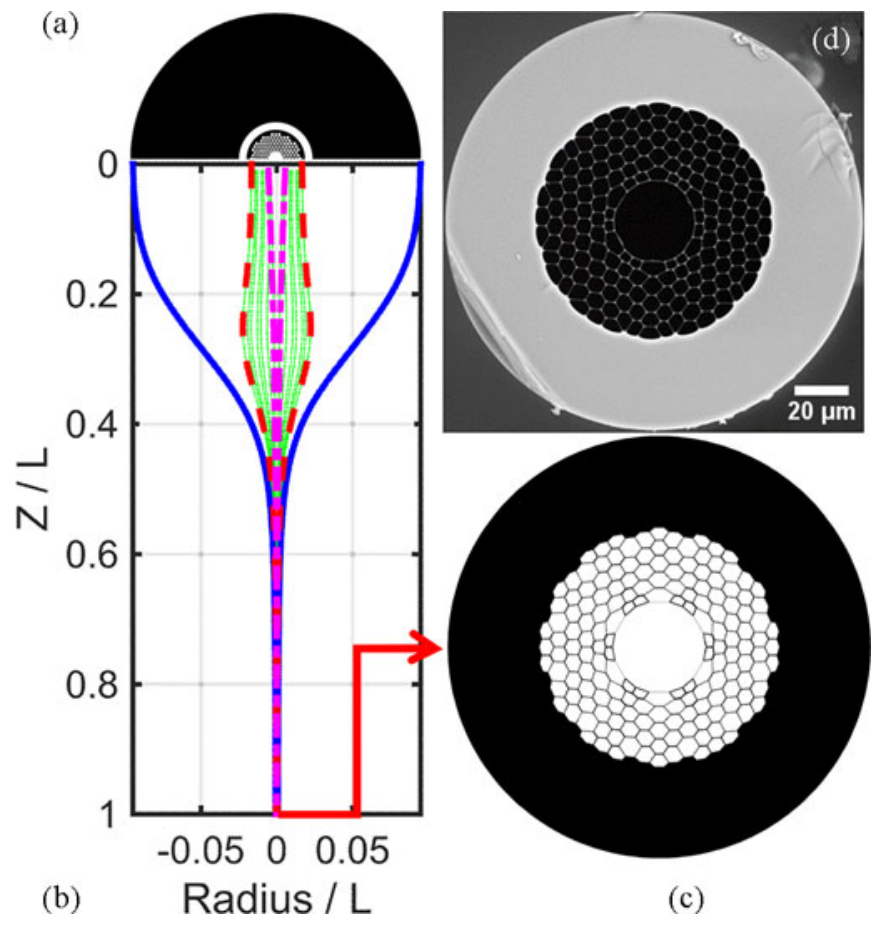

Fig. 3. (a) Simulated second stage preform; (b) Geometry evolution through the simulated draw-down process; (c) Simulated cross section of the final HCPBGF; (d) SEM image of the fabricated $1.55 \mu \mathrm{m}$ fiber.

e.g., to HC-PBGFs operating at shorter wavelengths. A scanning electron microscope (SEM) image of the fiber is shown in Fig. 1(a). The fiber has an outer diameter of $195 \mu \mathrm{m}$. The microstructured cladding region and hollow core are approximately 109 and $38 \mu \mathrm{m}$ in diameter, respectively. The average hole-to-hole spacing, $\Lambda$, and relative hole size, $\mathrm{d} / \Lambda$, were estimated to be $7.8 \mu \mathrm{m}$ and 0.991 , respectively.

During the draw, we also monitored in real-time the fiber outer diameter deviation from target in order to gain quantitative information on the stability of the drawing process and thus an indirect indication about the longitudinal uniformity of the fiber. The recorded trace is showed in Fig. 1(b). Over the full length, 
the root mean square (RMS) of the diameter deviation is just $0.3 \mu \mathrm{m}(\approx 0.16 \% \mathrm{RMS}, \approx 0.6 \%$ peak-to-peak $)$, which indicates excellent draw stability.

The length-averaged loss of the fiber was measured by performing a cutback measurement with a broadband source (we made separate measurements using both a supercontinuum laser and an incandescent white light source). A large mode area fiber was used at launch in order to minimize excitation of lossier higher order modes in the HC-PBGF. The spectral attenuation obtained from a $\approx 3.85 \mathrm{~km}$ cutback is shown in Fig. 2. From the plot the wide extent of the bandgap $(\approx 1550-2175 \mathrm{~nm})$, which correlates with the high air filling fraction $(>96 \%)$ of the fiber, is immediately apparent. The bandgap is segmented in three low loss regions as a result of the presence of residual surface modes which we ascribe to small scale deformations and asymmetries in the core surround [21]. The widest of the three regions is centered at about $2050 \mathrm{~nm}$ and contains the region of minimum loss, $3.2 \mathrm{~dB} / \mathrm{km}$ at $1984 \mathrm{~nm}$, and is over $160 \mathrm{~nm}$ wide. These loss and BW values are comparable to those reported previously [11], however in a $\approx 3.3 \times$ shorter span of fiber.

Though the relatively low loss value and low value of diameter deviation strongly suggest that the fiber is of consistent high quality over the full length, it would be ideal to get a direct experimental substantiation of this. However, suitable characterization tools, such as Optical Time Domain Reflectometers (OTDRs), are not well developed at the wavelength of $2 \mu \mathrm{m}$, so in the next phase of work targeting longer lengths of HC-PBGFs we switched to making fibers operating at the more conventional wavelength of $1.55 \mu \mathrm{m}$ where a far greater array of diagnostic tools are available.

\section{B. Fabrication of an $11 \mathrm{~km}$ Span of HC-PBGF at $1.55 \mu \mathrm{m}$}

A HC-PBGF operating at $\approx 1.55 \mu \mathrm{m}$ was obtained by suitably reducing the overall scale of the HC-PBGF whilst targeting a value of air filling fraction similar to that of the $2 \mu \mathrm{m}$ fiber. In this instance, through further fabrication and control improvements (discussed below), and partly owing to the reduced scale factor of the fiber itself, we obtained an overall yield of uniform fiber in excess of $11 \mathrm{~km}$ in a single fiber draw.

The fiber's structural parameters were obtained from high resolution SEM images, where particular care was devoted to minimizing the effect of the gold coating deposited on the samples. The fiber [Fig. 3(d)] has an outer diameter of $\approx 153 \mu \mathrm{m}$, $\approx 30 \mu \mathrm{m}$ core diameter, $6.2 \mu \mathrm{m}$ hole-to-hole spacing, a relative hole size $(\mathrm{d} / \Lambda)$ of 0.992 and an average cladding strut thickness of just $\approx 50 \mathrm{~nm}$. When considering the fiber length $(\approx 11 \mathrm{~km})$, we believe that some of the glass membranes in the cladding of this fiber have one of the most extreme aspect ratios ever demonstrated in an engineered material. Note that previous reports in the literature of HC-PBGFs fabricated with high values of air filling factor similar to our fiber were characterized by much shorter lengths [22] and/or by high loss [23], [24].

We employed optimization prior to fiber drawing using a novel fluid-dynamic model [18], capable of reproducing the complex HC-PBGF drawing process, Fig. 3(a)-(c), in association with improved control of hole pressurization, in order to

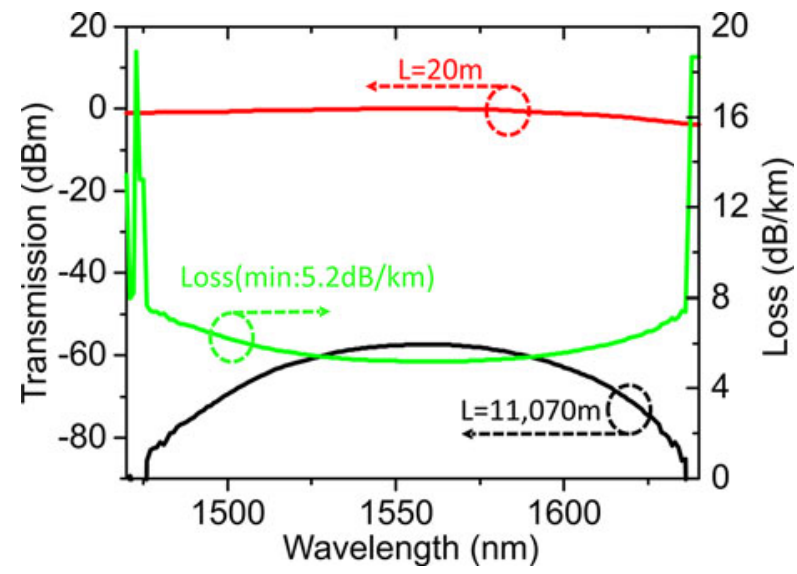

Fig. 4. Spectral attenuation of the full length of the HC-PBGF operating at $1.55 \mu \mathrm{m}$ (green curve), measured from an $11.05 \mathrm{~km}$ cutback, plotted with the associated cutback transmission data.

minimize any distortions of the core surround - a known problem at high expansion values that can lead to increased scattering loss [25] and to instability during the fiber draw.

The spectral attenuation of the fiber was obtained from cutback measurements using the same procedure described above for the $2 \mu \mathrm{m}$ HC-PBGF and a cutback length of $11.05 \mathrm{~km}$ (see Fig. 4). Over the measured wavelength range (1475-1635 nm, limited by OSA dynamic range), the plot shows a very flat loss profile with a minimum value of $5.2 \mathrm{~dB} / \mathrm{km}$ at $1560 \mathrm{~nm}$. Measurements on a shorter cutback length $(0.8 \mathrm{~km})$ provided a value for the $3 \mathrm{~dB}$ transmission BW of just over $200 \mathrm{~nm}$, which is to the best of our knowledge the widest ever reported in a 19 cell HC-PBGF. We have experimentally observed the low loss region of transmission to be largely insensitive to bending down to $15-20 \mathrm{~mm}$ radii, with some indication of increased loss at the edges likely due to surface mode interaction, similar to what we previously observed [26]. We note that the availability of long lengths of uniform HC-PBGF will enable the more complex characterization of microbending effects in these fibers in due course.

We investigated in detail the longitudinal consistency of this fiber. Fig. 5(a) displays the relative deviation from target diameter, measured as the fiber was drawn, demonstrating a small value $(\approx 0.24 \mu \mathrm{m}$, or $\approx 0.15 \% \mathrm{RMS}, \approx 0.7 \%$ peak-to-peak) over the whole $11 \mathrm{~km}$ length - similar to that observed for the $2 \mu \mathrm{m}$ HC-PBGF. We also used a high resolution OTDR to gain information on the longitudinal distribution of the loss along the fiber itself. However, as can be seen form Fig. 5(b), due to its relatively narrow dynamic range (e.g., $\approx 12-13 \mathrm{~dB}$ at the maximum spatial resolution of $\approx 0.5-1 \mathrm{~m}$ ), the OTDR can only provide information from a length up to $\approx 2 \mathrm{~km}$ from each end, but is totally unable to probe the central section of the HC-PBGF.

Therefore, we devised a different method to directly characterize the length-resolved transmission loss of our HC-PBGF. It consists of coupling light from an amplified diode laser $(1560 \mathrm{~nm})$ into the fiber and measuring the total out-scattered power as the fiber is wound at a constant speed through an integrating sphere fitted with an InGaAs detector-we term this technique Optical Side Scattering Radiometry (OSSR) [27]. In a 


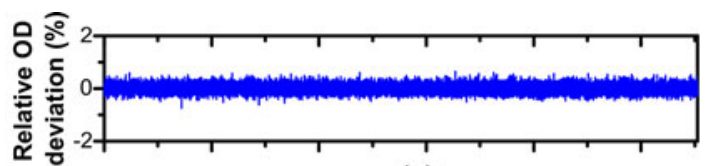

(a)

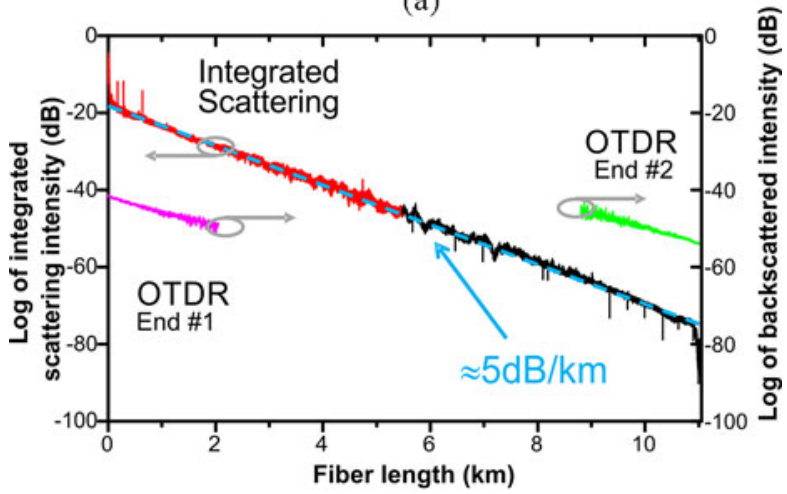

(b)

Fig. 5. (a) Diameter deviation versus fiber length measured during fiber drawing; (b) Integrated out-scattered intensity versus length, measured at the wavelength of minimum loss $(1560 \mathrm{~nm})$ : the red and black traces are measurements obtained from opposite ends of the $11 \mathrm{~km}$ span. Note the uniform $\approx 5 \mathrm{~dB} / \mathrm{km}$ slope, in excellent agreement with the cutback loss value. OTDR measurements are also shown (pink and green traces, plotted with an arbitrary offset for clarity), highlighting the shorter measurement reach and less accurate loss estimate $(\approx 4 \mathrm{~dB} / \mathrm{km})$. Note that the black and green traces are plotted reversed for graphic purposes.

normal scenario, the out-scattered power is observed to decrease at the same rate as the fiber loss. For validation, we measured a sample of Corning SMF-28e fiber and observed a linear slope (in $\mathrm{dB}$ scale) matching the loss value specified by the manufacturer. Any deviations from a linear slope indicates some form of longitudinal anomaly of the fiber structure, causing a loss increase (localized or distributed) and/or an increased coupling between the optical modes supported by the fiber.

In our HC-PBGF, we observed the scattered power to decrease at a constant rate of $5.14 \mathrm{~dB} / \mathrm{km}$, as shown in Fig. 5(b), in excellent agreement with the length-averaged loss value measured via cutback. To the best of our knowledge this is the first report of a detailed investigation of length dependent loss in a long HC-PBGF sample using an integrated out-scattered power method. The OSSR method provides for a far greater dynamic range $(\approx 60 \mathrm{~dB})$ than OTDR (equating to longer measurable lengths) and it can detect any localized fiber defects with far better spatial resolution $(\approx 10 \mathrm{~cm})$. Furthermore, it can be easily adapted to other wavelength ranges where it may be the easiest option for characterizing the longitudinal properties of fibers. Superimposed on the linear decrease in scattered power, a small number of discrete scattering peaks can be discerned, see red and black traces in Fig. 5(b). We ascribe such peaks to localized small-scale residual inconsistencies within the microstructure. The excess loss due to a single peak can be calculated by integrating the out-scattered power at the defective point and comparing it with that of an adjacent length of structurally consistent fiber. By using this procedure (explained more in detail in reference [27]) we estimated that the peaks account for a comparatively small excess loss contribution $(<1 \mathrm{~dB}$ out of $\approx 57 \mathrm{~dB}$

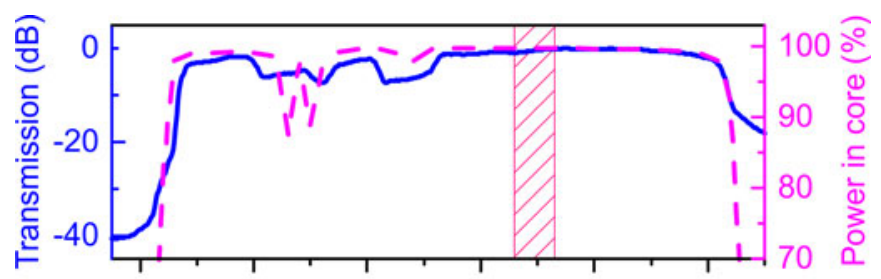

(a)

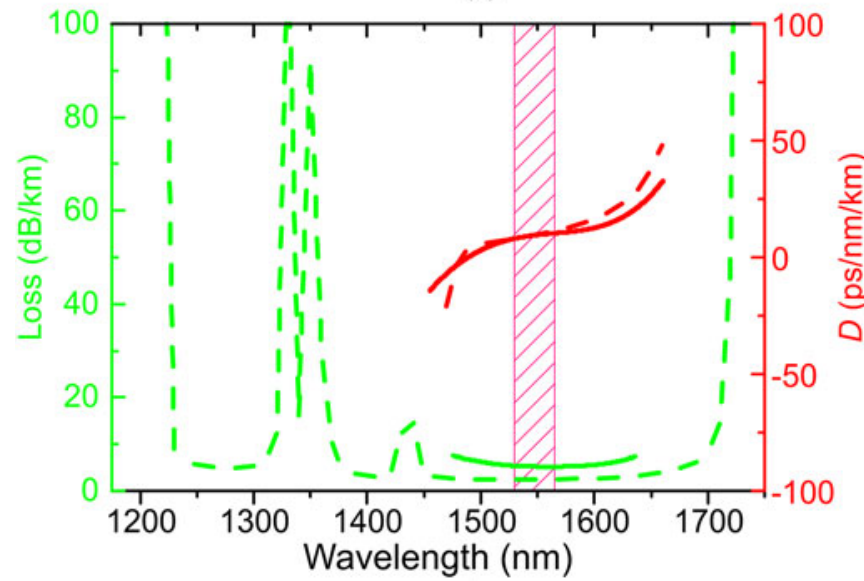

(b)

Fig. 6. (a) Comparison of the measured transmission of a $20 \mathrm{~m}$ length of HCPBGF (blue solid curve) with the calculated power in the core (magenta dashed), demonstrating excellent agreement of the position of the bandgap edges and of the surface mode anti-crossings. (b) Measured loss (green solid curve) and GVD (red) versus wavelength and the respective modelled (dashed) curves. The shaded area represents the wavelength range of the telecom C-band at $1.55 \mu \mathrm{m}$.

measured for the full fiber length). The integrated out-scattering measurement thus points to a high longitudinal consistency of the $11 \mathrm{~km}$ long HC-PBGF.

We also performed finite element simulations based on a model [28] that accurately reproduces the fiber geometry from high resolution SEM images. The model provides predictions for all the main transmission properties of the HC-PBGF, and a comparison with experimental data is shown in Fig. 6(a), (b). We observe that the model can accurately predict the position of the bandgap edges and that of the main surface mode groups within the photonic bandgap, see Fig. 6(a) and green traces in Fig. 6(b). A comparison between the modelled wavelength dependent group velocity dispersion (GVD) of the fundamental mode is also in good agreement with data obtained using low coherence interferometry (red traces). On the other hand, the model predicts a minimum loss value of $\approx 2.4 \mathrm{~dB} / \mathrm{km}$, thus about half of that experimentally measured in our fiber. Given that our scattering measurements indicate that the fiber is structurally very uniform, we conclude that other potential effects are likely responsible for the aforementioned loss discrepancy. These include, but are not limited to, mode coupling, micro bending effects, or a potential surface roughness increase with decreasing thickness of the glass struts. Whilst investigations are ongoing, the current fiber still exhibits an extremely good loss value and there are good prospects for further loss reduction once the cause of the discrepancy is identified and resolved. 
TABLE II

SUMMARY OF BEST PERFORMING HC-PBGFS

\begin{tabular}{lccccc}
\hline \hline Reference & Year & $\lambda(\mathrm{nm})$ & Loss $(\mathrm{dB} / \mathrm{km})$ & BW $(\mathrm{nm})$ & Length $(\mathrm{km})$ \\
\hline Mangan et al. $[13]$ & 2004 & 1565 & 1.7 & 20 & Up to 3 \\
Wheeler et al. [21] & 2012 & 1510 & 3.5 & 160 & 0.25 \\
Frosz et al. $[29]$ & 2013 & 1530 & 1.8 & 40 & 0.5 \\
van Uden et al. $[14]$ & 2014 & 1510 & 8 & 160 & 0.95 \\
Zhang et al. $[11]$ & 2015 & 1990 & 2.8 & 85 & 1.15 \\
Liu et al. $[19]$ & 2015 & 2010 & 3.2 & 160 & 3.85 \\
Mangan et al. $[30]$ & 2015 & 1538 & 6.5 & 12 & 2.75 \\
This work & $\mathbf{2 0 1 5}$ & $\mathbf{1 5 6 0}$ & $\mathbf{5 . 2}$ & $\mathbf{2 0 0}$ & $\mathbf{1 1 . 0 7}$ \\
\hline \hline
\end{tabular}

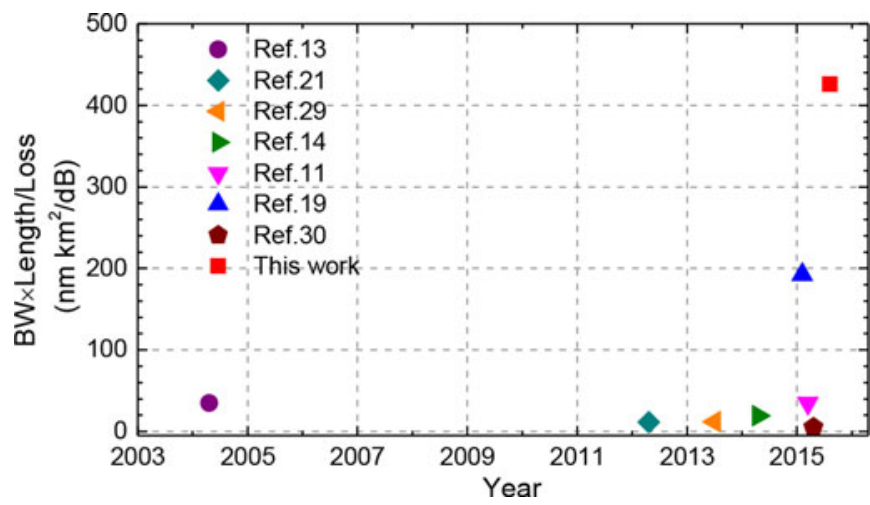

Fig. 7. Best performing HCPBGFs reported to date (summarized in the table) are compared via a length $\times \mathrm{BW} /$ loss figure of merit to visualize recent improvements.

\section{Performance Improvement and Comparison With the SOTA}

Table II presents a summary of the best performing HCPBGFs previously reported by groups worldwide, which are compared in terms of minimum loss, transmission BW and fabricated length to those discussed in the present study. In Fig. 7 we also plot the quantity length $\times \mathrm{BW} /$ loss as a figure of merit providing a visual indication of how the different fibers compare and a temporal perspective of the most recent advances obtained particularly in increasing the BW and the fiber length. The $11 \mathrm{~km}$ long HC-PBGF presented in this work provides a $\approx 20 \%$ increase in $\mathrm{BW}$ and at least a $\approx 4 \times$ increase in length as compared to the previous SOTA. Our models based on realistic fiber structures indicate that further significant loss reduction (substantially below $1 \mathrm{~dB} / \mathrm{km}$ ) should be possible [1]; furthermore, recent results obtained from a fluid dynamic model of the fiber drawing process confidently predict that it should be possible to scale up current fabrication to multi-hundred km lengths [17].

\section{DATA TRANSMISSION EXPERIMENTS}

We were able to demonstrate that both the fibers reported in this work were able to support error-free data transmission. In particular, use of the $3.85 \mathrm{~km}$ long span of $2 \mu \mathrm{m}$ HC-PBGF was demonstrated for single channel transmission at $52 \mathrm{Gbit} / \mathrm{s}$. A detailed description of this experiment has recently been published elsewhere [19]. In this section, we will focus on the results obtained using the $11 \mathrm{~km}$ long span of $1.55 \mu \mathrm{m}$ HC-PBGF.

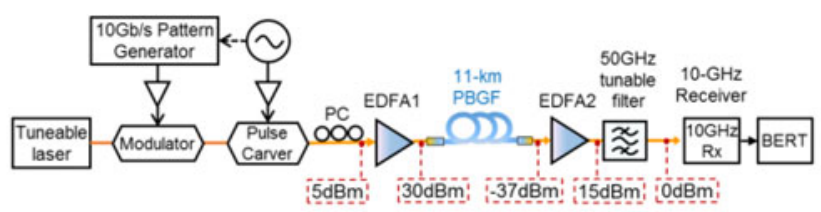

(a)

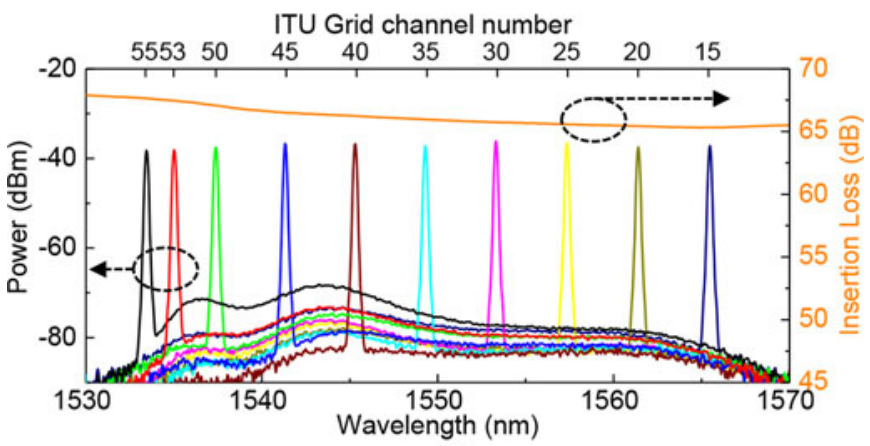

(b)

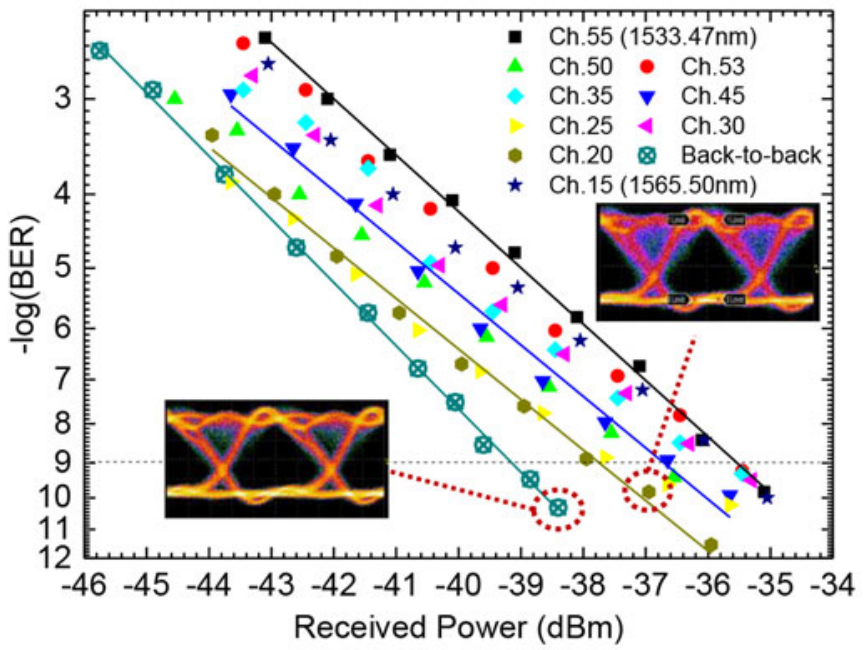

(c)

Fig. 8. (a) Experimental RZ-OOK data transmission setup; (b) Channels transmitted through the $11 \mathrm{~km} \mathrm{HC}-\mathrm{PBGF}$ ( $0.1 \mathrm{~nm}$ resolution); (c) BER measurements for back-to-back and various transmitted channels, and corresponding eye diagrams for back-to-back and channel 20.

As shown in Fig. 6, the width of the low loss region of the HC-PBGF $(\approx 200 \mathrm{~nm})$ significantly exceeds that of the telecom C-band. Thus, our demonstration was limited by the BW of the available optical amplifiers. The total insertion loss of the HC-PBGF span, including splice loss from interconnections to SMF 28 (and associated mode mismatch loss) was a substantial 66-67 dB. Such a high span loss required amplification of the signal to $\approx 30 \mathrm{dBm}$ prior to the transmission. Whilst use of such high power levels could cause serious drawbacks in long spans of conventional SMF, it was not expected to cause any penalty in the HC-PBGF due to the ultra-low nonlinearity of the HC-PBGF. We successfully tested both coherent transmission (QPSK) and direct detection using OOK.

We first show the results of direct detection as it allows for the lowest transmission latency, thus underlining this key feature of HC-PBGFs. Further, we used a return-to-zero modulation format as it allowed for better performance. The received signal 


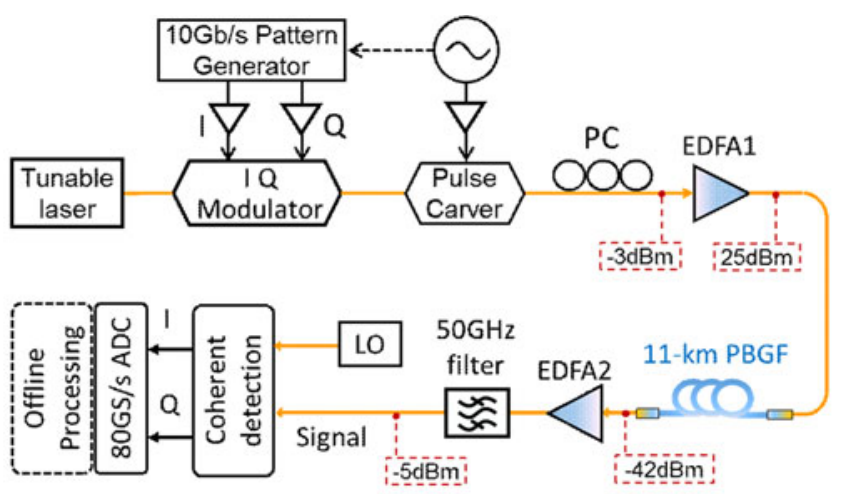

(a)

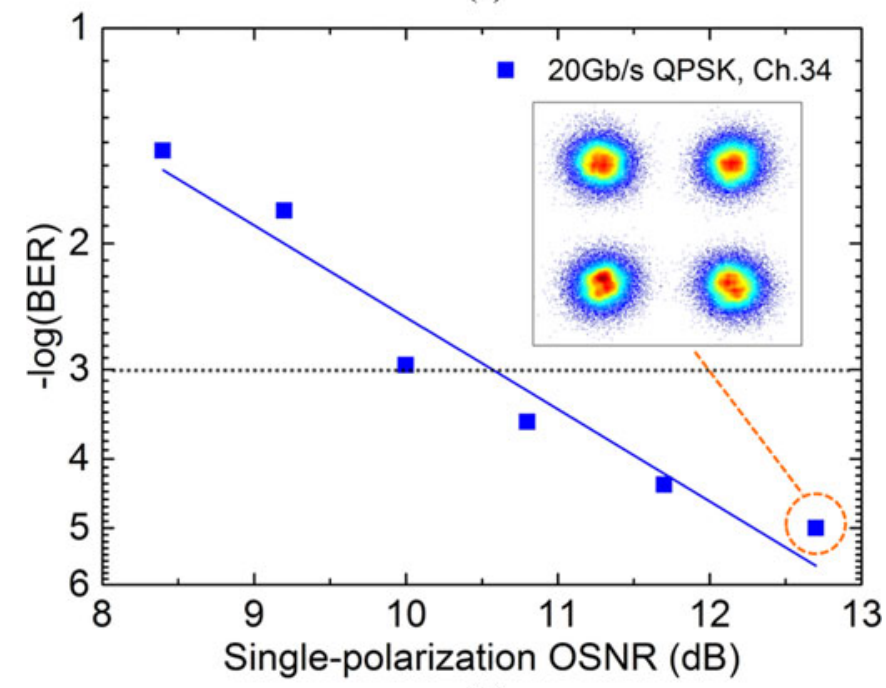

(b)

Fig. 9. (a) Experimental RZ-QPSK data coherent transmission setup; (b) BER measurements for the transmitted signal on channel $34(1550.12 \mathrm{~nm})$, and the constellation diagram of the demodulated signal at OSNR of $12.7 \mathrm{~dB}$.

$(\approx-37 \mathrm{dBm})$ was optically pre-amplified, filtered and detected with a 10-Gbit/s receiver, see Fig. 8(a). The optical spectra of several tested C-band channels (15 to 55) measured at the HCPBGF output end are shown in Fig. 8(b). Here, we notice that the optical signal-to-noise ratio (OSNR) in the region of 1537$1565 \mathrm{~nm}$ is almost constant and over $40 \mathrm{~dB}$. For wavelengths at the edges of the EDFA's gain BW (1532-1537 nm, 1565$1568 \mathrm{~nm})$, the OSNR and thus the transmission performance were slightly degraded due to ASE (Amplified Spontaneous Emission) from the input EDFA.

The Bit Error Ratio (BER) measurements are shown in Fig. 8(c). Wavelength channels in the flat-gain region have a BER performance close to that of the back-to-back (penalty $\leq 2 \mathrm{~dB}$ ). A penalty of up to $4 \mathrm{~dB}$ was measured for the transmission channels at the edges of the EDFA gain profile, which we believe is entirely due to the worse OSNR. Thus, we conclude that the fiber provides a similar performance across the entire $\mathrm{C}$-band with a few $\mathrm{dB}$ variation that is attributed to the fiber loss rather than any other effect (e.g., signal degradation due to modal crosstalk).

In addition to the direct detection experiment, we also tested the HC-PBGF using $20 \mathrm{~Gb} / \mathrm{s}$ QPSK coherent transmission as shown in Fig. 9(a). The experimental setup is similar to the direct detection experiment except for the use of an IQ modulator for QPSK generation and a coherent receiver with $80 \mathrm{GSa} / \mathrm{s}$ analogto-digital convertor for reception. The electronic data source used was a dual-channel arbitrary waveform generator (AWG, Tektronix AWG7122C) operating at $10 \mathrm{GSa} / \mathrm{s}$.

The BER as a function of single-polarization OSNR for channel $34(1550.12 \mathrm{~nm})$ is shown in Fig. 9(b). Error free transmission was achieved by launching $>25 \mathrm{dBm}$ power into the HC-PBGF (5 dB lower than that used for direct detection), which corresponded to a $>13 \mathrm{~dB}$ OSNR after the receiver-side pre-amplifier. The linear relationship of OSNR and $-\log (\mathrm{BER})$ confirms that the performance was limited by the fiber loss as was for the previously discussed direct detection experiment.

\section{CONCLuSions}

We have presented two record length HC-PBGFs operating in the 1.55 and $2 \mu \mathrm{m}$ spectral regions, respectively. Both fibers have loss of the order of a few $\mathrm{dB} / \mathrm{km}$ and very broad transmission bandwidths. The HC-PBGF operating at $2 \mu \mathrm{m}$ is $3.85 \mathrm{~km}$ long and has $\approx 3 \mathrm{~dB} / \mathrm{km}$ minimum loss and over $160 \mathrm{~nm} 3 \mathrm{~dB}$ transmission BW. Furthermore, a fiber operating at standard telecom wavelengths $(1.55 \mu \mathrm{m})$ was obtained in an unprecedented length of $11 \mathrm{~km}$. The length-averaged fiber loss, measured over the full $11 \mathrm{~km}$ span, was $\approx 5 \mathrm{~dB} / \mathrm{km}$ and the $3 \mathrm{~dB}$ transmission $\mathrm{BW}$ was found to be about $200 \mathrm{~nm}$ - a further improvement over HCPBGFs previously reported at this wavelength. An integrated side scattering technique was applied to measure the longitudinal distribution of the loss along the full fiber length, providing a first insight into the properties of such a long span of $\mathrm{HC}$ PBGF and convincing evidence of its excellent uniformity and structural consistency. Data transmission at $10 \mathrm{~Gb} / \mathrm{s}$ (direct detection) and $20 \mathrm{Gbit} / \mathrm{s}$ (coherent detection) along an $11 \mathrm{~km}$ span was demonstrated for the latter fiber, showing only penalties due to the fiber loss and achieving an estimated $>15 \mu$ s latency reduction relative to standard fiber.

The availability of long lengths of high performance $\mathrm{HC}$ PBGF with uniform transmission characteristics opens up the possibility of application trials of realistic scale. Furthermore, our numerical models of the fiber draw process give us confidence that much longer fiber yield per preform should be possible with further scaling of the process [17], and that much lower loss fibers should ultimately be possible.

\section{ACKNOWLEDGMENT}

The data for this paper can be found at doi: $10.5258 / \mathrm{SO}-$ TON/378704.

\section{REFERENCES}

[1] F. Poletti, M. N. Petrovich, and D. J. Richardson, "Hollow-core photonic bandgap fibers: Technology and applications," Nanophotonics, vol. 2, no. 6, pp. 315-340, 2013.

[2] F. Poletti, N. V. Wheeler, M. N. Petrovich, N. K. Baddela, E. Numkam Fokoua, J. R. Hayes, D. R. Gray, Z. Li, R. Slavík, and D. J. Richardson, "Towards high-capacity fibre-optic communications at the speed of light in vacuum," Nature Photon., vol. 7, no. 4, pp. 279-284, 2013. 
[3] X. Peng, M. Mielke, and T. Booth, "High average power, high energy $1.55 \mu \mathrm{m}$ ultra-short pulse laser beam delivery using large mode area hollow core photonic band-gap fiber," Opt. Exp., vol. 19, no. 2, pp. $923-$ 932, 2011.

[4] R. Slavík, G. Marra, E. Numkam Fokuoa, N. Baddela, N. V. Wheeler, M. Petrovich, F. Poletti, and D. J. Richardson, "Ultralow thermal sensitivity of phase and propagation delay in hollow core optical fibres," Sci. Rep., 2015 , to be published.

[5] L. Olanterä, C. Sigaud, J. Troska, F. Vasey, M. N. Petrovich, F. Poletti, N. V. Wheeler, J. P. Wooler, and D. J. Richardson, "Gamma irradiation of minimal latency Hollow-Core Photonic Bandgap Fibres," J. Instrum., vol. 8, art. no. C12010, 2013.

[6] V. A. Sleiffer, Y. Jung, P. Leoni, M. Kuschnerov, N. V. Wheeler, N. K. Baddela, R. Van Uden, C. Okonkwo, J. R. Hayes, J. P. Wooler, E. R. Numkam Fokoua, R. Slavik, F. Poletti, M. N. Petrovich, V. Veljanovski, S. U. Alam, D. J. Richardson, and H. De Waardt, " $30.7 \mathrm{~Tb} / \mathrm{s}(96 \times 320$ Gb/s) DP-32QAM transmission over 19-cell Photonic Band Gap Fiber," presented at the Optical Fiber Communication Conf., Anaheim, CA, USA, 2013, paper OW1I.5.

[7] V. Sleiffer, Y. Jung, N. K. Baddela, J. Surof, M. Kuschnerov, V. Veljanovski, J. R. Hayes, N. V. Wheeler, E. Numkam Fokoua, J. P. Wooler, D. R. Gray, N. Wong, F. Parmigiani, S. U. Alam, M. N. Petrovich, F. Poletti, D. J. Richardson, and H. De Waardt, "High capacity mode-division multiplexed optical transmission in a novel 37-cell hollow-core photonic bandgap fiber," J. Lightw. Technol., vol. 32, no. 4, pp. 854-863, 2014.

[8] S. R. Sandoghchi, G. T. Jasion, N. V. Wheeler, S. Jain, Z. Lian, J. P. Wooler, R. P. Boardman, N. K. Baddela, Y. Chen, J. R. Hayes, E. Numkam Fokoua, T. Bradley, D. R. Gray, S. M. Mousavi, M. N. Petrovich, F. Poletti, and D. J. Richardson, "X-ray tomography for structural analysis of microstructured and multimaterial optical fibers and preforms," Opt. Exp., vol. 22, no. 21, pp. 26181-26192, 2014.

[9] J. K. Lyngs $\varnothing$, B. J. Mangan, C. Jakobsen, and P. J. Roberts, "7-cell core hollow-core photonic crystal fibers with low loss in the spectral region around $2 \mu \mathrm{m}$," Opt. Exp., vol. 17, no. 26, pp. 23468-23473, 2009.

[10] Y. Chen, N. V. Wheeler, N. Baddela, J. Hayes, S. R. Sandoghchi, E. Numkam Fokoua, M. Li, F. Poletti, M. Petrovich, and D. J. Richardson, "Understanding wavelength scaling in 19-cell core hollow-core photonic bandgap fibers," presented at the Optical Fiber Communication Conf., San Francisco, CA, USA, 2014, paper M2F.4.

[11] H. Zhang, N. Kavanagh, Z. Li, J. Zhao, N. Ye, Y. Chen, N. V. Wheeler, J. P. Wooler, J. R. Hayes, S. R. Sandoghchi, F. Poletti, M. N. Petrovich, S. U. Alam, R. Phelan, J. O'Carroll, B. Kelly, L. Grüner-Nielsen, D. J. Richardson, B. Corbett, and F. C. Garcia Gunning, "100 Gbit/s WDM transmission at $2 \mu \mathrm{m}$ : Transmission studies in both low-loss hollow core photonic bandgap fiber and solid core fiber," Opt. Exp., vol. 23, no. 4, pp. 4946-4951, 2015.

[12] Z. Li, A. M. Heidt, N. Simakov, Y. Jung, J. M. O. Daniel, S. U. Alam, and D. J. Richardson, "Diode-pumped wideband thulium-doped fiber amplifiers for optical communications in the $1800-2050 \mathrm{~nm}$ window," Opt. Exp., vol. 21, no. 22, pp. 26450-26455, 2013.

[13] B. Mangan, L. Farr, A. Langford, P. J. Roberts, D. P. Williams, F. Couny, M. Lawman, M. Mason, S. Coupland, R. Flea, H. Sabert, T. A. Birks, J. C. Knight, and R. S. J. Philip, "Low loss $(1.7 \mathrm{~dB} / \mathrm{km})$ hollow core photonic bandgap fiber," presented at the Optical Fiber Communication Conf., Los Angeles, CA, USA, 2004, paper PD24.

[14] R. Van Uden, C. Okonkwo, H. Chen, N. V. Wheeler, F. Poletti, M. Petrovich, D. J. Richardson, H. De Waardt, and A. Koonen, "8.96Tb/s (32 $\times 28$ GBaud $\times 32$ QAM) transmission over $0.95 \mathrm{~km} 19$ cell hollow-core photonic bandgap fiber," presented at the Optical Fiber Communication Conf., 2014, San Francisco, CA, USA, paper W4J.3.

[15] S. R. Sandoghchi, T. Zhang, J. P. Wooler, N. Baddela, N. V. Wheeler, Y. Chen, G. T. Jasion, D. R. Gray, E. Numkam Fokoua, J. Hayes, M. Petrovich, F. Poletti, and D. J. Richardson, "First investigation of longitudinal defects in hollow core photonic bandgap fibers," presented at the Optical Fiber Communication Conf., San Francisco, CA, USA, 2014, paper M2F.6.

[16] Y. Chen and T. A. Birks, "Predicting hole sizes after fibre drawing without knowing the viscosity," Opt. Mater. Exp., vol. 3, no. 3, 346-356, 2013.

[17] G. T. Jasion, F. Poletti, J. Shrimpton, and D. J. Richardson, "Volume manufacturing of hollow core photonic band gap fibers: challenges and opportunities," presented at the Optical Fiber Communication Conf., Los Angeles, CA, USA, 2015, paper W2A.37.

[18] G. T. Jasion, J. S. Shrimpton, Y. Chen, T. Bradley, D. J. Richardson, and F. Poletti, "MicroStructure element method (MSEM): Viscous flow model for the virtual draw of microstructured optical fibers," Opt. Exp., vol. 23, no. 1, pp. 312-329, 2015.

[19] Z. Liu, Y. Chen, Z. Li, B. Kelly, R. Phelan, J. O'Carroll, T. Bradley, J. P. Wooler, N. V. Wheeler, A. M. Heidt, T. Richter, C. Schubert, M. Becker, F. Poletti, M. N. Petrovich, S. U. Alam, D. J. Richardson, and R. Slavík, "High-capacity directly modulated optical transmitter for $2-\mu \mathrm{m}$ spectral region," J. Lightw. Technol., vol. 33, no. 7, pp. 1373-1379, Apr. 2015.

[20] Y. Chen, Z. Liu, S. R. Sandoghchi, G. T. Jasion, T. Bradley, E. Numkam Fokoua, J. Hayes, N. V. Wheeler, D. R. Gray, B. J. Mangan, R. Slavik, F. Poletti, M. N. Petrovich, and D. J. Richardson, "Demonstration of an $11 \mathrm{~km}$ hollow core photonic bandgap fiber for broadband low-latency data transmission," presented at the Optical Fiber Communication Conf. Post Deadline Papers, Los Angeles, CA, USA, 2015, paper Th5A.1.

[21] N. V. Wheeler, M. N. Petrovich, R. Slavik, N. K. Baddela, E. R. Numkam Fokoua, J. R. Hayes, D. R. Gray, F. Poletti, and D. J. Richardson, "Widebandwidth, low-loss, 19-cell hollow core photonic band gap fiber and its potential for low latency data transmission," presented at the Nat. Fiber Optic Engineering Conf., Los Angeles, CA, USA, 2012, paper PDP5A.2.

[22] R. Amezcua-Correa, F. Gèrôme, S. G. Leon-Saval, N. G. R. Broderick, T. A. Birks, and J. C. Knight, "Control of surface modes in low loss hollowcore photonic bandgap fibers," Opt. Exp., vol. 16, no. 2, pp. 1142-1149, 2008.

[23] L. Dong, B. K. Thomas, S. Suzuki, and L. Fu, "Extending transmission bandwidth of air-core photonic bandgap fibers," Opt. Fiber Technol., vol. 16, no. 6, pp. 442-448, 2010.

[24] P. S. Light, F. Couny, Y. Wang, N. V. Wheeler, P. J. Roberts, and F. Benabid, "Double photonic bandgap hollow-core photonic crystal fiber," Opt. Exp., vol. 17, no. 18, pp. 16238-16243, 2009.

[25] E. N. Fokoua, D. J. Richardson, and F. Poletti, "Impact of structural distortions on the performance of hollow-core photonic bandgap fibers," Opt. Exp., vol. 22, no. 3, pp. 2735-2744, 2014.

[26] Y. Jung, V. A. J. M. Sleiffer, N. K. Baddela, M. N. Petrovich, J. R. Hayes, N. V. Wheeler, D. R. Gray, E. R. N. Fokoua, J. Wooler, N. Wong, F. Parmigiani, S. Alam, J. Surof, M. Kuschnerov, V. Veljanovski, H. De Waardt, F. Poletti, and D. J. Richardson, "First demonstration of a broadband 37-cell hollow core photonic bandgap fiber and its application to high capacity mode division multiplexing," presented at the Optical Fiber Communication Conf., Anaheim, CA, USA, 2013, paper PDP5A.3.

[27] S. R. Sandoghchi, D. R. Gray, Y. Chen, N. V. Wheeler, T. Bradley, J Hayes, E. Numkam Fokoua, G. T. Jasion, S. M. Mousavi, M. Petrovich, F. Poletti, and D. J. Richardson, "High dynamic range technique for discrete and distributed scattering loss measurement in microstructured optical fibres," in Proc. Eur. Conf. Opt. Commun., 2015, paper Mo.3.1.4.

[28] E. R. Numkam Fokoua, S. R. Sandoghchi, Y. Chen, N. V. Wheeler, N. K Baddela, J. R. Hayes, M. N. Petrovich, D. J. Richardson, and F. Poletti, "Accurate modelling of hollow core photonic bandgap fibre," presented at the Advanced Photonics Conf., Barcelona, Spain, 2014, paper SoM4B.4.

[29] M. H. Frosz, J. Nold, T. Weiss, A. Stefani, F. Babic, S. Rammler, and P. St J. Russell, "Five-ring hollow-core photonic crystal fiber with $1.8 \mathrm{~dB} / \mathrm{km}$ loss," Opt. Lett., vol. 38, no. 13, pp. 2215-2217, 2013.

[30] B. J. Mangan, M. Kuschnerov, J. W. Nicholson, J. Fini, L. Meng, R. Windeler, E. Monberg, A. Desantolo, and V. Mikhailov, "First demonstration of hollow-core fiber for intra data center low latency connectivity with a commercial $100 \mathrm{gb} / \mathrm{s}$ interface," presented at the Optical Fiber Communication Conf., Los Angeles, CA, USA, 2015, paper M3D.4.

Yong Chen received the Ph.D. degree in physics from the University of Bath, Bath, U.K., in 2013. Since then, he has been with the Optoelectronics Research Centre, University of Southampton, Southampton, U.K., as a Research Fellow, mainly working on the development of low-loss, broad-bandwidth, and long-length hollow-core photonic bandgap fibers. His research interests include design, fabrication, and characterization of microstructured optical fibers, postprocessing of conventional and microstructured fibers, mode converters, and nonlinear fiber optics.

Dr. Chen is a Member of the Optical Society of America. 
Zhixin Liu (S'07-M'12) received the B.Eng. degree in information engineering and the B.B.A. degree in business administration from Tianjin University, Tianjin, China, in 2006, the M.S. degree in electrical engineering from Shanghai Jiao Tong University, Shanghai, China, in 2009, and the Ph.D. degree in information engineering from the Chinese University of Hong Kong, Hong Kong, in 2012. Since 2013, he has been with the Optoelectronics Research Centre, University of Southampton, Southampton, U.K. His current research includes high-speed direct modulation and coherent optical signal processing with focus on phase locking, advance modulation formats, advanced multiplexing/demultiplexing schemes, and their system applications.

Seyed Reza Sandoghchi (S'11) received the Bachelor's degree (Hons.) in telecommunication engineering from the Sadjad Institute of Higher Education, Mashhad, Iran, in 2005. In 2011, he received the Master's of engineering degree in optical telecommunication from the University of Malaya, Kuala Lumpur, Malaysia, and is currently working toward the Ph.D. degree in the Optoelectronics Research Centre, University of Southampton. He has a strong background in electromagnetism, numerical modeling, and optical fiber fabrication. His research interest includes among other microscopy and nanoscopy, and fiber characterization methods. His current research focuses on hollow core photonic band gap fibers, where his main research activities include developing characterization systems.

Gregory T. Jasion received the M.Eng. degree in aerospace engineering from the University of Southampton, Southampton, U.K. Following by the Ph.D. degree from the University of Southampton on the topic of Helicopter blown dust, in the field of multiphase flow with an emphasis on computational modeling. These interests brought him to the Optoelectronics Research Centre to develop fluid dynamics models to improve the fabrication process of hollow-core photonic band gap fibers.

Thomas D. Bradley received the M.Phys. and Ph.D. degrees from the University of Bath, Bath, U.K., in 2009 and 2014, respectively. His Ph.D. degree and earlier career research has focused on the fabrication, characterization, and loading of gas species in both anti-resonant and photonic bandgap hollow core fibers. He joined the Optoelectronics Research Centre in 2014, where he is currently a Postdoctoral Researcher in the micro-structured optical fiber groups. His current research interests include the fabrication of low loss anti-resonant hollow core fibers, development of novel characterization tools for optical fibers and gas filling in hollow core fibers. In 2014, he received the Best Early Career Presentation Prize at the EPSRC Manufacturing the Future conference.

Eric Numkam Fokoua received the B.Sc. degree in microelectronics from Nankai University, Tianjin, China, in 2008, followed by the M.Sc. degrees in photonics from the Universities of St. Andrews, Scotland and Ghent, Belgium, in 2010. Having joined the Optoelectronics Research Centre soon after, he received the $\mathrm{Ph}$.D. degree in optoelectronics in 2014 for work on the numerical modeling of hollow core photonics bandgap fibers. His research interests include numerical simulations of dielectric optical waveguides and their applications.
John Hayes received the Ph.D. degree from the ORC for his development of highly innovative micro-structured fibers. He joined the Optoelectronics Research Centre (ORC) in 2003 from industry, where he was with Pirelli developing the vapor axial deposition (VAD) method for the high volume manufacture of single-mode telecoms fibers. He was part of a team that initially transferred VAD technology from Sumitomo Electric Industries in Japan and established a U.K. fiber manufacturing facility, and later became the Head in a team of engineers that supported its $24 / 7$ operation. Since joining the ORC, he has created a diverse range of novel microstructured fibers. He is currently a Senior Experimental Officer.
Natalie V. Wheeler received the Ph.D. degree from the University of Bath, Bath, U.K., in 2011, on the fabrication and integration of hollow core photonic crystal fibers (HC-PCFs) for use as gas cells in applications including frequency metrology and slow light. In 2010, he started as a Research Fellow in the ORC at the University of Southampton, Southampton, U.K., working on the fabrication of HC-PCFs for telecommunications applications, designing and fabricating $\mathrm{HC}-\mathrm{PCF}$ which have been used in world-leading transmission experiments which highlight the potential of HC-PCFs in future high-capacity transmission networks. She has co-authored more than 90 journal and conference papers and was recently received a prestigious Royal Society University Research Fellowship. Her current research interests also include fabrication of low loss and low bend sensitivity HC-PCFs for operation in the mid-IR and gas spectroscopy.

David R. Gray was born in Maidstone, U.K. He received the M.Phys. degree in physics with photonics from the University of Southampton, Southampton, U.K., in 2011, and is currently working toward the Ph.D. degree working on characterization techniques of multimode optical fibers at the Optoelectronics Research Centre, University of Southampton. His research interests include fiber characterization, measurement automation, and digital signal processing. $\mathrm{He}$ is a Member of the Optical Society of America.

Brian J. Mangan, biography not available at the time of publication.

Radan Slavík (M’07-SM'07) received the M.Sc. and Ph.D. degrees in optics and optoelectronics from the Faculty of Mathematics and Physics, Charles University, Prague, Czech Republic, in 1996 and 2000, respectively, and the D.Sc. degree from the Academy of Sciences of the Czech Republic, Praha, Czech Republic, in 2009. In 1995-2000 and 2004-2009, he was with the Institute of Photonics and Electronics, Czech Academy of Sciences, Prague. In 2000-2003, he was with the Centre d'Optique, Photonique et Laser, Universite Laval, Quebec, Canada, as a Postdoctoral Research Fellow. Since 2009, he has been with the Optoelectronics Research Centre, University of Southampton, Southampton, U.K. His research interests focus on optical and optics-assisted signal processing.

Dr. Slavík is a member of the Optical Society of America. 
Francesco Poletti is currently the holder of a Royal Society University Fellowship and a Principal Research Fellow at the Optoelectronics Research Centre (ORC), University of Southampton, Southampton, U.K. He has worked for three years on optical network design at Marconi Communications and for more than ten years at the ORC developing new generations of microstructured optical fibers. His research interests include the design of photonic bandgap and antiresonant fibers, the development of fiber-optic characterization techniques, and the study of nonlinear optical effects in novel fibers. He has co-authored more than 60 journal and 160 conference publications and is a frequent invited speaker at major international conferences in optics.

Marco N. Petrovich (M'11-SM'12) received the Laurea degree in physics from the University of Padua, Padua, Italy, in 1998, and the Ph.D. degree in optoelectronics engineering from the University of Southampton, Southampton, U.K., in 2003. He joined the Optoelectronics Research Centre (ORC) in 1999 as the recipient of a prestigious Ph.D. studentship funded by Pirelli Cables. He has been since involved in multiple aspects of fiber fabrication. In 2001, he received the A.E. Owen prize of the Society of Glass Technology, U.K., for his work on chalcogenide fibers. From 2003, he has been working on silica microstructured fibers. He is currently a Principal Research Fellow at the ORC and a Member of the EPSRC Centre for Innovative Manufacturing in Photonics. His research interests include the development of novel fiber structures, including photonic bandgap, antiresonant, highly nonlinear, dispersion tailored fibers and their application in telecommunications, gas sensing, and power delivery.

Dr. Petrovich is a Senior Member of the Optical Society of America, has co-authored more than 190 publications in journals and conference proceedings and is a frequent Invited Speaker at international conferences and meetings. He serves in the technical program committee of the IEEE Sensors conference and is an Associate Editor of the IEEE SENSORS JOURNAL.
David J. Richardson (F'14) received the B.Sc. and Ph.D. degrees in fundamental physics from Sussex University, Brighton, U.K., in 1985 and 1989, respectively. He joined the Optoelectronics Research Centre (ORC), Southampton University, Southampton, U.K., in 1989, and received a Royal Society University Fellowship in 1991 in recognition of his pioneering work on short pulsed fiber lasers. He is currently a Deputy Director in the ORC with responsibility for the ORC's fiber related activities. His current research interests include among others: optical fiber communications, microstructured optical fibers, and pulsed high-power fiber lasers. He is a prominent figure in the international photonics community and has published more than 900 conference and journal papers and produced more than 20 patents.

Prof. Richardson is a Fellow of the Optical Society of America and the Institute of Engineering and Technology. He became a Fellow of the Royal Academy of Engineering in 2009. 